\title{
Pullulan-based nanoparticles: future therapeutic applications in transmucosal protein delivery
}

\author{
"The analysis of all the data available in the literature suggests the adequacy of the biomaterial pullulan for drug-delivery \\ strategies. Particularly, although counting on a reduced number of works, pullulan-based nanoparticles reveal potential \\ regarding transmucosal protein delivery."
}

\section{Keywords: nanoparticles $=$ proteins $=$ pullulan $\|$ transmucosal delivery}

Current advances in the field of drug delivery are aimed at finding adequate strategies for the administration of different drugs. Transmucosal delivery is the first-line option for the systemic delivery of many drugs, including proteins, a group of molecules with recognized therapeutic potential and wide range of applications (drug and genetic therapy, immunization, among others). This strategy, however, demands developing adequate carriers, as proteins are very sensitive molecules, easily undergoing degradation in physiological media, mainly due to $\mathrm{pH}$ and enzymatic occurrences. Polymeric nanoparticles have taken the forefront of this carrierdesign challenge, offering appealing properties that include a reduced size providing intimate contact with mucosal surfaces, which potentiates any active action of the carrier regarding transmucosal delivery. Nanoparticles have been generally demonstrated to improve protein pharmacokinetic profiles, not only by providing increased stabilization but, in some cases, also permitting controlled release and enhancing drug absorption [1]. Moreover, the high surface-to-volume ratio displayed by these carriers increases drug loading capacity [2]. Polysaccharides have been indicated for many years as the most promising materials in drug carrier development, namely regarding nanoparticles. In this regard, a particular emphasis is placed on chitosan. Other polysaccharides, however, have been paving their way, also demonstrating great ability for protein delivery. Pullulan is one of those polysaccharides; the first works reporting the application of pullulan-based nanoparticles for drug delivery dating back to 1998 , when a cholesteryl group bearing pullulan was used to produce nanoparticles for the delivery of both an oncoprotein [3] and insulin [4].
Pullulan is a neutral, water-soluble polysaccharide produced from starch by the fungus Aureobasidium pullulans, by means of a process of fermentation [5]. Chemically, this polymer consists of $\alpha-(1,6)$-linked maltotriose residues, which in turn are composed of three glucose molecules connected to each other by $\alpha-(1,4)$ glycosidic bonds [6,7]. Several properties have been reported for this material that make it attractive for drug-delivery purposes, including its adhesive ability, as well as the capacity to form fibers and thin biodegradable films [7]. The high content of hydroxyl groups on pullulan chains further endows the polymer with inherent physiological activity and also provides chemical flexibility.

Biodegradability is a mandatory characteristic of any material being considered for an application in drug delivery, and pullulan is expected to comply with it. Actually, once administered it will be exposed to the hydrolysis of glycoside bonds and the subsequent metabolism of glucose [8]. The ability to adhere to cell surfaces might also be advantageous within the application, improving the carriers' residence time and, consequently, the potential for timely absorption of the encapsulated protein. In this regard, apart from the demonstrated role of pullulan on the adhesion of $A$. pullulans to biological surfaces, such as leaves [9], cell adhesion to pullulan-based surfaces was reported on a number of occasions [10-12]. Pullulan-based nanoparticles were actually reported to adhere to the nasal epithelium in a study regarding nasal vaccination [13], and the authors' group has also verified the adherence of pullulan nanoparticles to respiratory epithelial cells (Calu-3 and A549), although to a limited extent [Rodrigues S, Unpublished Data].

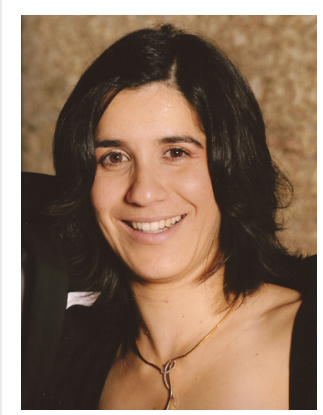

\section{Ana Grenha}

Author for correspondence: University of Algarve, Centre for Molecular \& Structural Biomedicine/Institute for Biotechnology \& Bioengineering, Faculty of Sciences \& Technology, Campus de Gambelas,

8005-139 Faro, Portugal Tel.: +351289800100 Fax: +351 289818419

E-mail: amgrenha@ualg.pt

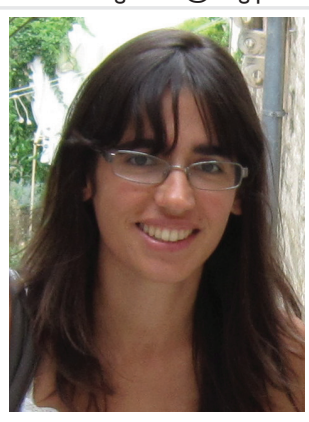

Susana Rodrigues University of Algarve, Centre for Molecular \& Structural Biomedicine/Institute for Biotechnology \& Bioengineering, Faculty of Sciences \& Technology, Campus de Gambelas, Portugal

\section{FUTURE SCIENCE}


Pullulan has been increasingly described as matrix-forming material of drug and gene nanocarriers [5,7,13-17]. The most common characteristic of these carriers is the use of hydrophobic derivatives of pullulan, namely cholesteryl-pullulan, because this provides an amphiphilic polymer with the capacity to form nanoparticles by self-aggregation. These nanoparticles have been demonstrated to form stable complexes with both hydrophobic and hydrophilic drugs, such as proteins, reinforcing their flexibility. In a remarkable detail, apart from the capacity to form nanocarriers with suitable properties for transmucosal protein delivery, it has also been reported that pullulan-based nanoparticles are able to preserve the structure of encapsulated proteins, thus ensuring their biological activity [4]. Nevertheless, although the potential application in protein delivery was mentioned frequently, the demonstration of results in the area has been scarce. The delivery of insulin, bovine serum albumin, DNA and IL-12 was effectively proposed [4,15-18]. The authors' group has recently given a contribution to the field. Instead of using a hydrophobic derivative of pullulan, our strategy involved the synthesis of charged hydrophilic derivatives of the polymer that provided the possibility of establishing electrostatic interactions among them, or with other charged materials of interest. From the synthesized derivatives (carboxylated, phosphorylated, aminated and sulfated) the latter two were used to produce polysaccharide-based nanoparticles, upon complexation with either chitosan or carrageenan, or even with each other. The carriers were produced using a mild technique of polyelectrolyte complexation, which only involves hydrophilic conditions and a simple mixture of solutions, while permitting a very rapid formation of the carriers (less than $10 \mathrm{~min}$ ). These nanoparticles exhibited great ability for the association of proteins with distinct properties (insulin and bovine serum albumin, MW of 5.7 and $67 \mathrm{kDa}$, respectively) and were proposed for nasal and pulmonary transmucosal delivery. Having demonstrated a clear non-cytotoxic behavior in model respiratory cell lines (Calu-3 and A549) [17], the nanoparticles also revealed to not induce an inflammatory response upon contact with the same cell lines [Rodrigues S, Unpublished Data]. Finally, preliminary in vivo tests in rats demonstrated an increased therapeutic response of insulin-loaded pullulan-based nanoparticles administered intranasally as compared with an insulin solution of similar dose [Grenha A, Unpublished Data].

The analysis of all the data available in the literature suggests the adequacy of the biomaterial pullulan for drug-delivery strategies. Particularly, although counting on a reduced number of works, pullulan-based nanoparticles reveal potential regarding transmucosal protein delivery. The ability to associate and release biomacromolecules is demonstrated, good indications are available concerning biocompatibility, and biodegradability is also ensured. However, the real possibilities of success should be considered on a rational and cautious basis. Looking at chitosan nanoparticles, for instance, which are incomparably more explored and for a longer time, after more than two decades of research and really good, diverse and important demonstrations of effectiveness and safety, no formulation has been made commercially available so far. Nanomedicine is still a controversial matter, mainly because of safety issues. The regulatory agencies demand uncountable studies and demonstrations to consider any approval, which is highly desirable if considering that global human health is the addressed subject. However, it is natural that nanomedicine, as a recent subject in the ambit of regulation, faces even more difficult challenges.

\section{"The real possibilities of success should be considered on a rational and cautious basis. Looking at chitosan nanoparticles, for instance, which are incomparably more explored and for a longer time ... no formulation has been made commercially available so far."}

As a demonstration that nanomedicine is still in its infancy regarding the regulatory challenges to be faced, it is interesting to consider a work from 2012 that revealed the significant accumulation of nanoparticles $(40-350 \mathrm{~nm})$ in the ovaries [19]. When other organs like the lung, liver, spleen and kidneys are usually examined to determine the biodistribution of nanoparticles and, thus, evaluate potential toxicity, the ovaries seem to have been neglected. The observation of that work raises an extremely important question: although in vitro cell studies give very relevant indications on the biocompatibility behavior of nanocarriers, it is 
becoming undeniable that a complete characterization of potential toxicity is only possible with early in vivo studies. For this reason, keeping track of the whole biological portrait and the complex interactions between the nanocarrier and the biological structures, which might induce toxicological issues, is essential when designing a nanodelivery system.

It seems, then, that the potential of pullulanbased nanoparticles for transmucosal protein delivery is there, but a very long way is still ahead to cover.

\section{Financial \& competing interests disclosure}

This work was supported by national Portuguese funding through Fundação para a Ciência e a Tecnologia, project reference PTDC/SAU-FCF/100291/2008, PTDC/DTPFTO/0094/2012 and PEst-OE/EQB/LA0023/2013. The authors have no other relevant affiliations or financial involvement with any organization or entity with a financial interest in or financial conflict with the subject matter or materials discussed in the manuscript apart from those disclosed.

No writing assistance was utilized in the production of this manuscript.

\section{References}

1 Grenha A. Chitosan nanoparticles: a survey of preparation methods. J. Drug Target. 20(4), 291-300 (2012).

2 De La Fuente M, Csaba N, Garcia-Fuentes M, Alonso MJ. Nanoparticles as protein and gene carriers to mucosal surfaces. Nanomedicine 3, 845-857 (2008).

3 Gu X, Schmitt M, Hiasa A et al. A novel hydrophobized polysaccharide/oncoprotein complex vaccine induces in vitro and in vitro cellular and humoral immune responses against HER2-expressing murine sarcomas. Cancer Res. 58(15) 3385-3390 (1998).

4 Akiyoshi K, Kobayashi S, Shichibe S et al. Self-assembled hydrogel nanoparticle of cholesterol-bearing pullulan as a carrier of protein drugs: complexation and stabilization of insulin. J. Control. Release 54(3), 313-320 (1998).

5 Rekha M, Chandra P. Pullulan as a promising biomaterial for biomedical applications: a perspective. Trends Biomater. Artif. Organs 20(2), 000-000 (2007).

6 Mizrahy S, Peer D. Polysaccharides as building blocks for nanotherapeutics. Chem. Soc. Rev. 41, 2623-2640 (2012).

7 Cheng K-C, Demirci A, Catchmark J. Pullulan: biosynthesis, production, and applications. Appl. Microbiol. Biotechnol. 92(1), 29-44 (2011).

8 Teramoto N, Shibata M. Synthesis and properties of pullulan acetate. Thermal properties, biodegradability, and a semi-clear gel formation in organic solvents. Carbohydr. Polym. 63(4), 476-481 (2006).

9 Pouliot JM, Walton I, Nolen-Parkhouse M, Abu-Lail LI, Camesano TA. Adhesion of aureobasidium pullulans is controlled by uronic acid based polymers and pullulan. Biomacromolecules 6(2), 1122-1131 (2005).

10 Shi L, Aid R, Le Visage C, Chew SY. Biomimicking polysaccharide nanofibers promote vascular phenotypes: a potential application for vascular tissue engineering. Macromol. Biosci. 12(3), 395-401 (2012).

11 Autissier A, Letourneur D, Le Visage C. Pullulan-based hydrogel for smooth muscle cell culture. J. Biomed. Mater. Res. A 82(2), 336-342 (2007).

$12 \mathrm{Na}$ K, Shin D, Yun K, Park KH, Lee KC. Conjugation of heparin into carboxylated pullulan derivatives as an extracellular matrix for endothelial cell culture. Biotechnol. Lett. 25(5), 381-385 (2003).

13 Nochi T, Yuki Y, Takahashi H et al. Nanogel antigenic protein-delivery system for adjuvant-free intranasal vaccines. Nat. Mater. 9(7), 572-578 (2010).

14 Jeong Y-I, Nah J-W, Na H-K et al. Selfassembling nanospheres of hydrophobized pullulans in water. Drug Dev. Ind. Pharm. 25(8), 917-927 (1999).

15 Gupta M, Gupta AK. Hydrogel pullulan nanoparticles encapsulating pBUDLacZ plasmid as an efficient gene delivery carrier. J. Control. Release 99(1), 157-166 (2004).

16 Shimizu T, Kishida T, Hasegawa U et al. Nanogel DDS enables sustained release of IL-12 for tumor immunotherapy. Biochem. Biophys. Res. Commun. 367(2), 330-335 (2008).

17 Dionísio M, Cordeiro C, Remuñán-López C, Seijo B, Rosa da Costa A, Grenha A. Pullulan-based nanoparticles as carriers for transmucosal protein delivery. Eur. J. Pharm. Sci. 50, 102-113 (2013).

18 Ayame H, Morimoto N, Akiyoshi K. Selfassembled cationic nanogels for intracellular protein delivery. Bioconjug. Chem. 19(4), 882-890 (2008).

19 Schädlich A, Hoffmann S, Mueller T et al. Accumulation of nanocarriers in the ovary: a neglected toxicity risk? J. Control. Release 160(1), 105-112 (2012). 